\title{
Anterior Tarsal Tunnel Syndrome With Thrombosed Dorsalis Pedis Artery: A Case Report
}

\author{
Naseemul Gani ${ }^{1}$; Hayat Ahmad Khan $^{1,{ }^{1,}}$; Younis Kamal $^{1}$; Munir Farooq ${ }^{1}$; Hina Jeelani ${ }^{1}$; Adil \\ Bashir Shah ${ }^{1}$ \\ ${ }^{1}$ Department of orthopedics, Govt hospital for bone and joint surgery Barzullah, Srinager, India \\ ${ }^{*}$ Corresponding author: Hayat Ahmad Khan, Department of orthopedics, Govt hospital for bone and joint surgery Barzullah, Srinager, India. Tell: +91-9906672626, Fax: +91-194- \\ 2423389, E-mail: drhayatkhan@gmail.com
}

Received: July 3, 2014; Revised: August 23, 2014; Accepted: December 28, 2014

\begin{abstract}
Introduction: The aanterior tarsal tunnel syndrome denotes the entrapment of the deep peroneal nerve under the inferior extensor retinaculum. Although various etiological factors have been reported to cause anterior tarsal syndrome, its occurrence with thrombosed dorsalis pedis artery has not been reported in the English literature.

Case Presentation: A 40 -year-old male patient was presented with the history of persistent pain along the dorsal surface of right foot, which was aggravated with the activities. Conservative management was tried without much relief. Diagnosis of anterior tarsal tunnel syndrome was made and the patient was planned for surgery. Thrombosed dorsalis pedis artery was found along with two adjacent collateral vessels. Retinaculum was released and nerve was mobilized. Tight compartment got released. Postoperative period was uneventful. No recurrence was seen on follow-up.

Conclusion: The anterior tarsal tunnel syndrome is a known disease. A high index of clinical suspicion is required while dealing with the chronic cases. A detailed history to rule out any traumatic event is necessary too. Timely investigations and surgical release give dramatic relief.
\end{abstract}

Keywords:Anterior Tarsal Tunnel Syndrome; Thrombosed artery; Tarsal Tunnel Syndrome

\section{Introduction}

The anterior tarsal tunnel syndrome denotes (ATTS) the entrapment of the deep peroneal nerve under the inferior extensor retinaculum. The usual complaints are dysesthesias in the first web space and opposing sides of the adjacent toes with or without proximally radiating dysesthesias. Clinical findings are decreased touch and pinprick in the first web space. Tinel's sign over the deep peroneal nerve beneath the inferior extensor retinaculum may be present and extensor digitorum brevis muscle may be atrophic. Mostly, it is seen in runners. Its etiology as described in literature can be due to tight shoes or ski boots, ganglion cyst, dorsal osteophytes at the ankle, midtarsal or metatarsocuneiform articulation. Although various etiological factors have been reported to cause anterior tarsal syndrome, its occurrence with thrombosed dorsalis pedis artery has not been reported in the literature to the best of our knowledge.

\section{Case Presentation}

A 40 -year-old male patient, nondiabetic, nonsmoker and normotensive was presented with the history of persistent pain along the dorsal surface of right foot, which was aggravated with the activities. Conservative manage- ment was tried for 8 months without much relief. Diagnosis of ATTS was made on clinical examination. Nerve Conduction velocity (NCV) showed decreased conduction in deep peroneal nerve and stress test was positive. Patient was planned for operation. Under spinal anesthesia, the body part was prepared and tourniquet inflated. Intraoperatively, thrombosed dorsalis pedis artery was found along with two adjacent collateral vessels (Figure 1, 2 , and 3). Retinaculum was released and nerve was mobilized. No adherence with the surrounding structures was found. Tight compartment got released. Upon deflating the tourniquet blood flow in the collaterals was checked, which was adequate. Dorsal pulsation was felt too. No excision was done. Wound was closed and the patient was followed up in outpatient department. Over 9 months of follow up, no recurrence of any symptoms was noted.

Tarsal tunnel corresponds to the carpel tunnel in the limb counterpart. Tight compartments exert pressure over the nerves resulting in the compressive neuropathies. Tarsal tunnel syndrome as the name suggests relates to the tightening of the inferior extensor retinaculum of the foot. It causes compression over the deep peroneal nerve resulting in the spectrum of symptoms. Numerous factors have been described to cause this compression. Intrinsic factors mentioned in the literature are 
Gani N et al.

due to osteophytes, ganglionic cysts or lipomas. These factors cause compensatory forefoot valgus leading to subtalar supination and midtarsal inversion leading to the pressure over the nerve.

Distally the nerve is vulnerable to injury due to lack of protective sheath. Extrinsic factors like tight shoe laces or the external trauma can cause injury to the nerve. Edema or fibrosis causes pressure over the nerve and neural ischemia. The relation of nerve with the tendons and the vascular structures is depicted in Figure 1. The little space in the tunnel formed by the inferior extensor retinaculum and the close proximity of the structures make the nerve vulnerable to compressive neuropathy. In an already small space, any addition to the structure can cause pressure over the nearby structures. The final brunt is to be burnt by the nerve, as happened in our case.

The patient had trivial trauma at the ankle, which can cause thrombosis at the dorsalis pedis artery. Over time, the collaterals had developed to compensate for the dysfunctional arterial segment and to bypass it. Addition of new vessels in the already tight tunnel had resulted in the pressure over the nearby structures, including the nerve. The mixed sensorimotor nature of the deep peroneal nerve leads to both sensory and motor symptoms as in our case.

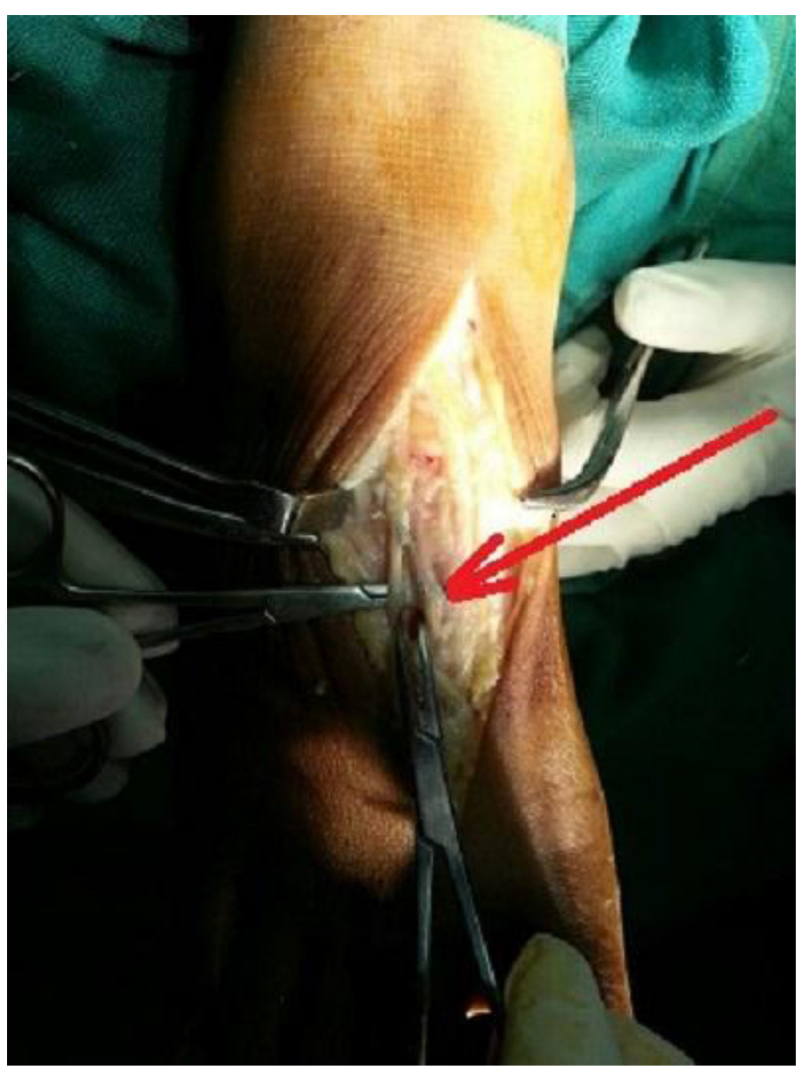

Figure 1. Thrombosed Segment of Dorsalis Pedis Artery

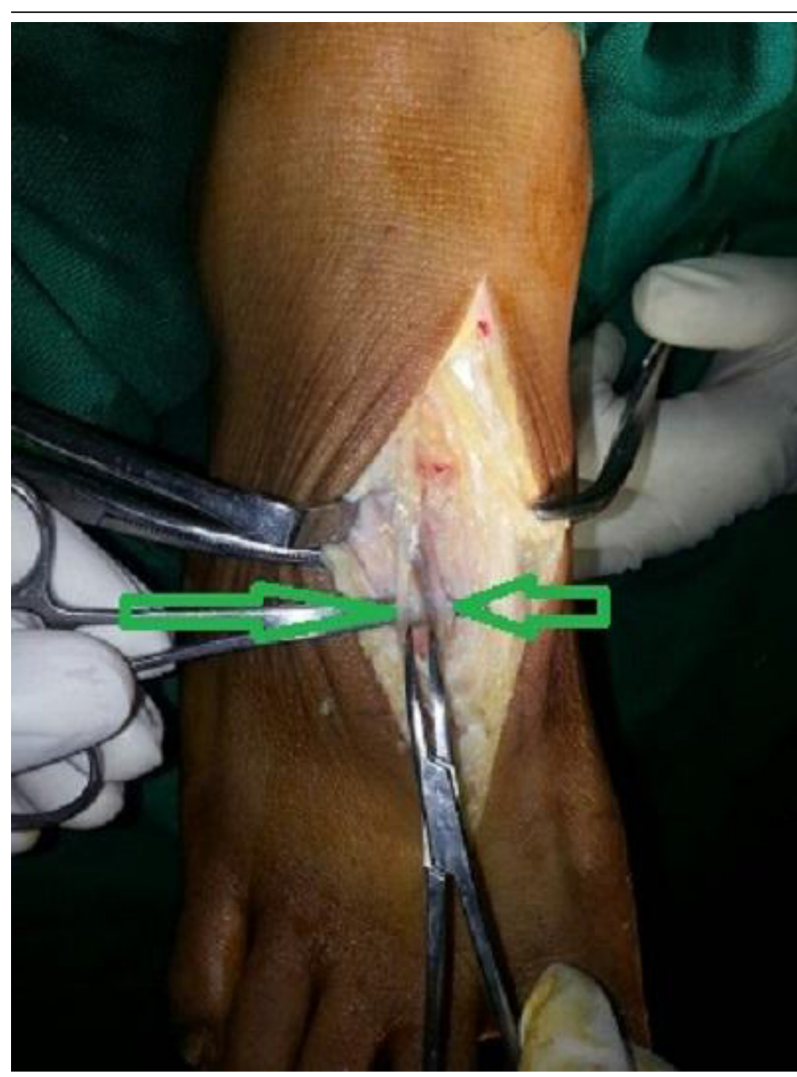

Figure 2. Well-Developed Collateral Vessels on the Sides of Thrombosed Segment

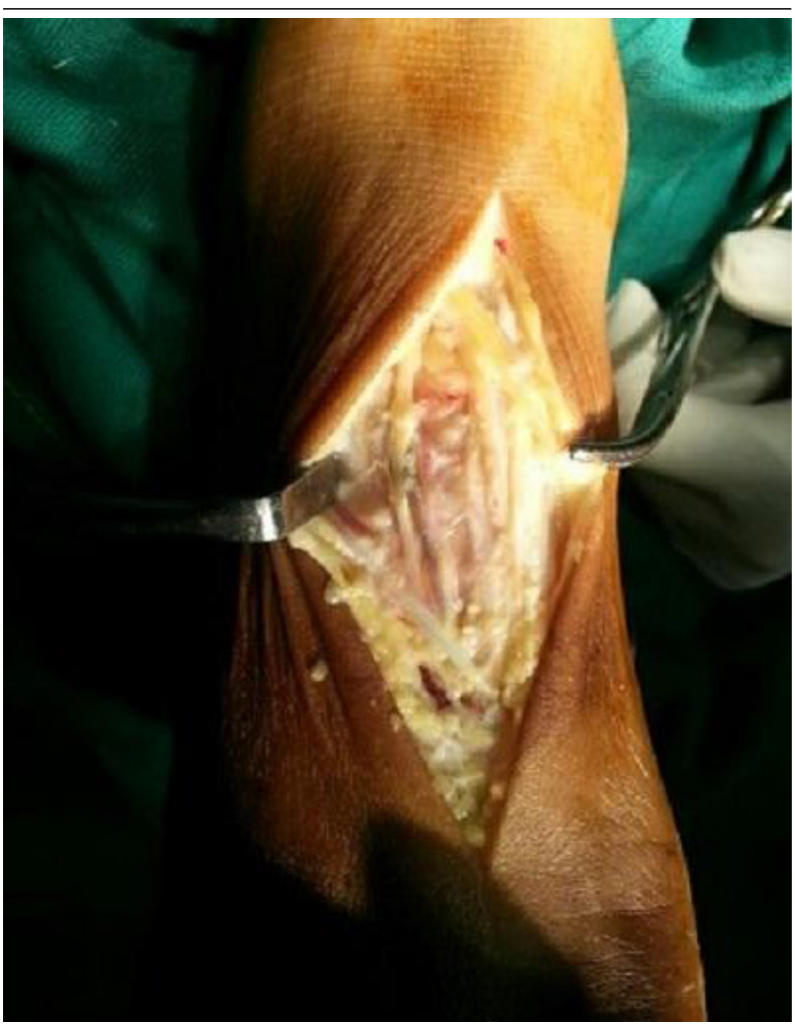

Figure 3. Released Extensor Retinaculum With Free Structures 
Gani N et al.

\section{Discussion}

Other possible mechanism was preexisting thrombosis, presented with the tarsal tunnel syndrome. The tight retinaculum has caused the thrombosis and over time collaterals had developed, which caused further pressure over the nerve. The cause effect phenomenon is difficult to ascertain and needs more pathophysiological studies.

The decompression because of release of inferior extensor retinaculum causes relief and no recurrence proves the pathobiomechanics. The well-formed collaterals have actually taken up from the parent artery and the distal oxygen saturation was $100 \%$ as was checked Perioperatively with the pulse oximetry.

On reviewing the literature, the posterior tibial nerve and the deep peroneal nerve compression have been described by the Keck (1) and Kopell et al. (2), respectively. The latter was called the anterior tarsal syndrome by Marinacci (3) years after the anatomy of the inferior extensor retinaculum was described by Romanes (4).

Possible etiological factors described in the literature are sprains, subluxations, or fracture fragments, high boots, high healed or tightly laced shoes which mentioned by Krause et al. (5) Gessini et al. (6). In ballet dancers, stretching of the nerve due to extreme planter flexion was described by Borges (7). Cetinkal et al. reported such a case in a missed talar fracture (8). Akyuz after evaluating 325 symptomatic feet found only 14 (4.3\%) patients who had ATTS (9). To the best of our knowledge, this is so far the largest series in the PubMed search engine. Although sporadic cases of anterior tarsal syndromes have been reported in literature since the inception of the term ATTS (10-13), its association with the thrombosed dorsalis pedis artery has not been documented.

Anterior tarsal tunnel syndrome is a known disease. A high index of clinical suspicion is required while dealing with the chronic cases. A detailed history to rule out any traumatic event is necessary. Timely investigations and surgical release give dramatic relief.

\section{References}

1. Keck C. The tarsal tunnel syndrome [database on the Internet]. 1962

2. Kopell HP, Thompson WAL. Peripheral entrapment neuropathies.Baltimore: Williams \& Wilkins; 1963.

3. Marinacci AA. Neurological syndromes of the tarsal tunnels. Bull Los Angeles Neurol Soc. 1968;33(2):90-100.

4. Romanes GJe. Cunningham's Textbook of Anatomy. Med Educ $J . ; \mathbf{4 0}(1)$.

5. Krause $\mathrm{KH}$, Witt T, Ross A. The anterior tarsal tunnel syndrome. J Neurol.1977;217(1):67-74

6. Gessini L, Jandolo B, Pietrangeli A. The anterior tarsal syndrome. Report of four cases. J Bone Joint Surg Am. 1984;66(5):786-7.

7. Borges LF, Lawrence F, Mark Hallett MD, Dennis J, Selkoe MD, keasley Welsh K. The Anterior Tarsal Tunnel Syndrome Report of Two Cases. JNS. 1981;54(1):89-92.

8. Cetinkal A, Topuz K, Kaya S, Colak A, Demircan MN. Anterior tarsal tunnel syndrome secondary to missed talus fracture: a case report. Turk Neurosurg. 2011;21(2):259-63.

9. Akyuz G, Us O, Turan B, Kayhan O, Canbulat N, Yilmar IT. Anterior tarsal tunnel syndrome. Electromyogr Clin Neurophysiol. 2000;40(2):123-8.

10. Andresen BL, Wertsch JJ, Stewart WA. Anterior tarsal tunnel syndrome. Arch Phys Med Rehabil.1992;73(11):1112-7.

11. Koo YS, Cho CS, Kim BJ. Pitfalls in using electrophysiological studies to diagnose neuromuscular disorders. I Clin Neurol. 2012;8(1):1-14.

12. Kuruvilla A. Accessory deep peroneal nerve. Neurol India. 2004;52(1):135

13. Lindenbaum BL. Ski boot compression syndrome. Clin Orthop Relat Res.1979(140):109-10. 